\title{
CARACTERÍSTICAS ESTRUTURAIS DE PERFILHOS VEGETATIVOS E REPRODUTIVOS EM PASTOS DIFERIDOS DE CAPIM-BRAQUIÁRIA
}

\author{
Manoel Eduardo Rozalino Santos, ${ }^{1}$ Dilermando Miranda da Fonseca, ${ }^{2}$ Eric Márcio Balbino, ${ }^{3}$ \\ Simone Pedro da Silva, ${ }^{4}$ João Paulo Ismério dos Santos Monnerat ${ }^{4}$ e Virgilio Mesquita Gomes ${ }^{5}$
}

1. Doutorando em Zootecnia pela Universidade Federal de Viçosa na área de Forragicultura e Pastagens. E-mail: m_rozalino@yahoo.com.br

2. Professor do Departamento de Zootecnia da Universidade Federal de Viçosa

3. Doutorando em Zootecnia pela Universidade Federal de Viçosa na área de Bioclimatologia

4. Mestrandos em Zootecnia pela Universidade Federal de Viçosa na área de Nutrição de Ruminantes

5. Doutorando em Zootecnia pela Universidade Federal de Viçosa na área de Forragicultura e Pastagens.

\section{RESUMO}

Este trabalho foi desenvolvido com o objetivo de comparar as características estruturais de perfilhos vegetativos e reprodutivos em pastos de Brachiaria decumbens cv. Basilisk diferidos por 73, 95 e 116 dias. O delineamento experimental foi em blocos casualizados, com três repetições, no esquema de parcelas subdivididas. $\mathrm{O}$ período de diferimento não influenciou $(\mathrm{P}>0,10)$ o número de perfilhos vegetativos $(\mathrm{PV})$ e incrementou $(\mathrm{P}<0,10)$ o número de perfilhos reprodutivos (PR) (de 16 para 117 perfilhos $/ \mathrm{m}^{2}$ ). Em pastos diferidos por até 95 dias, $\mathrm{PR}$ foi mais pesado $(\mathrm{P}<0,05)$ do que PV. Contrariamente ao $\mathrm{PV}$, a relação entre número e peso de $P R$ foi positiva $(\mathrm{P}<0,10)$. Verificou-se incremento linear $(\mathrm{P}<0,01 \mathrm{e}$
$\mathrm{P}<0,10$ ) do comprimento do pseudocolmo dos perfilhos em função do período de diferimento da pastagem. O número de folhas vivas (NFV) por perfilho não foi incrementado $(\mathrm{P}>0,10)$ pelo período de diferimento. Os $\mathrm{PR}$ possuíram $(\mathrm{P}<0,05)$ maior comprimento do pseudocolmo, maior NFM e menor NFV do que o PV. Os PR possuíram maiores $(\mathrm{P}<0,05)$ percentuais de colmo e folha morta, bem como menor percentual de folha viva $(\mathrm{P}<0,05)$ do que os PV. O PV possui melhor composição morfológica do que o PR. O período de diferimento altera as características estruturais e piora a composição morfológica dos perfilhos em pastos diferidos de Brachiaria decumbens cv. Basilisk.

PALAVRAS-CHAVES: Brachiaria decumbens, composição morfológica, número de folhas, número de perfilho, peso de perfilho.

\section{ABSTRACT}

\section{STRUCTURAL CHARACTERISTICS OF VEGETATIVE AND REPRODUCTIVE TILLERS ON DIFERRED SIGNALGRASS} PASTURES

The aim of this work was to compare the structural characteristics of vegetative and reproductive tillers on Brachiaria decumbens $\mathrm{cv}$. Basilisk pastures diferred for 73, 95 and 116 days. Randomized block design in a split plot scheme with three replications was used. The stockpiling period did not influence $(\mathrm{P}>0.10)$ the number of vegetative tillers (VT) but increased $(\mathrm{P}<0.10)$ the number of reproductive tillers (RT) (from 16 to 117 tillers $/ \mathrm{m}^{2}$ ). On pastures diferred for up to 95 days, RT were heavier $(\mathrm{P}<0.05)$ than VT. Contrarily to VT, the relation between the number and weight of reproductive tillers was positive $(\mathrm{P}<0.10)$. A linear increase $(\mathrm{P}<0.01$ and $\mathrm{P}<0.10)$ for tiller pseudostem length in relation to the diferring periods of the pasture was found. The number of living leaves (NLL) per tiller was not increased $(\mathrm{P}>0.10)$ by the deferring period. The $\mathrm{RT}$ revealed $(\mathrm{P}<0.05)$ a higher pseudostem length, higher number of dead leaves (NDL) and lower NLL when compared to VT. RT showed $(\mathrm{P}<0.05)$ higher percentages of stem and dead leaf as well as a lower percentage of living leaf $(\mathrm{P}<0.05)$ than VT. VT showed a better morphological composition than RT. The deferring period alters the structural characteristics and worsens the morphological composition of tillers on deferred Brachiaria decumbens cv. Basilisk pastures.

KEYWORDS: Brachiaria decumbens, leaf number, morphological composition, tiller number, tiller weight. 


\section{INTRODUÇÃO}

O diferimento do uso da pastagem é estratégia de manejo eficaz e de fácil adoção, capaz de minimizar os problemas decorrentes da estacionalidade de produção das forrageiras tropicais. Espécies forrageiras de colmo delgado, com crescimento satisfatório durante o outono e perda de valor nutritivo menos intensa durante seu crescimento, são adequadas para o manejo sob pastejo diferido e, nesse sentido, o capim-braquiária (Brachiaria decumbens cv. Basilisk) destaca-se como opção adequada (EUCLIDES et al., 1990).

Os resultados de produção vegetal e animal, obtidos em condições de pastagens diferidas, dependem das ações de manejo empregadas, tal como a duração do período de diferimento da pastagem, que é um dos determinantes da quantidade e qualidade da forragem diferida, das possíveis perdas de forragem durante o pastejo e da estrutura do pasto diferido.

A estrutura do pasto diferido pode ser avaliada, dentre outros fatores, pela quantificação e caracterização das suas distintas categorias de perfilhos. Isso é verdade porque os perfilhos são as unidades modulares de crescimento da gramínea (HODGSON, 1990) e, dessa forma, o pasto pode ser considerado como uma população de perfilhos em diferentes estádios de desenvolvimento e, por conseguinte, com características estruturais diferentes.

Um dos critérios para a classificação dos perfilhos consiste na identificação do seu estádio de desenvolvimento (SANTOS et al., 2009b), o que possibilita distingui-los em vegetativos e reprodutivos. Em pastos diferidos essa classificação, quando aliada à quantificação dos perfilhos, é interessante porque permite inferir sobre outras características do pasto, tais como as massas de seus componentes morfológicos (SANTOS et al., 2010).

Salienta-se que uma mesma categoria de perfilho pode apresentar características variáveis em função da ação de manejo adotada no diferimento da pastagem, razão pela qual é necessária a determinação das características de perfilhos individuais para caracterização mais efetiva do pasto. Sendo o período de diferimento um dos principais fatores determinantes da forragem produzida, torna-se importante avaliar seu efeito sobre as características estruturais dos perfilhos, como alterações no número de folhas, no tamanho do colmo e na composição morfológica.
Este trabalho foi desenvolvido com o objetivo de classificar, quantificar e comparar as características estruturais de perfilhos em pastos de Brachiaria decumbens cv. Basilisk sob períodos de diferimento.

\section{MATERIAL E MÉTODOS}

No período de janeiro a julho de 2006, desenvolveu-se o experimento no Setor de Forragicultura do Departamento de Zootecnia da Universidade Federal de Viçosa, localizada em Viçosa, MG (20 ${ }^{\circ} 45^{\prime}$ 'S; $42^{\circ} 51^{\prime}$ $\mathrm{W} ; 651 \mathrm{~m}$ ), numa área de pastagem de Brachiaria decumbens cv. Basilisk (Stapt.), com $540 \mathrm{~m}^{2}$. O solo da área experimental é Latossolo Vermelho-Amarelo de textura argilosa. A análise química do solo, realizada no início do período experimental, na camada $0-20 \mathrm{~cm}$, apresentou os seguintes resultados: $\mathrm{pH} \mathrm{em} \mathrm{H}_{2} \mathrm{O}: 5,4$; $\mathrm{P}$ : 1,5 (Mehlich-1) e K: $115 \mathrm{mg} / \mathrm{dm}^{3} ; \mathrm{Ca}^{2+}: 1,8 ; \mathrm{Mg}^{2+}: 0,7$ $\mathrm{e} \mathrm{Al}^{3+}: 0,2 \mathrm{cmol}_{\mathrm{c}} / \mathrm{dm}^{3}(\mathrm{KCl} 1 \mathrm{~mol} / \mathrm{L})$. Durante o período de avaliação foram registrados dados climáticos em estação meteorológica distante da área experimental aproximadamente $500 \mathrm{~m}$ (Tabela 1).

O experimento seguiu delineamento em blocos casualizados com três repetições, em esquema de parcela subdividida. Foram avaliados três períodos de diferimento (73, 95 e 116 dias) e duas categorias de perfilhos (vegetativo e reprodutivo). Nas parcelas de $12,38 \mathrm{~m}^{2}$ considerou-se a área útil de $5,25 \mathrm{~m}^{2}$. Para a implementação das avaliações, as parcelas no pasto de capim-braquiária foram diferidas em épocas distintas, quais sejam: 7/3/2006, 28/3/2006 e 19/4/2006; e o período de diferimento encerrado em única data $(1 / 7 / 2006)$.

A altura dos pastos foi monitorada semanalmente e mantida em aproximadamente $20 \mathrm{~cm}$ até o início do diferimento. Para isso, adotou-se a técnica de mob-grazing. Efetuou-se a adubação fosfatada no dia 6 de janeiro de 2004, com a aplicação de $50 \mathrm{~kg} / \mathrm{ha}$ de $\mathrm{P}_{2} \mathrm{O}_{5}$, na forma de superfosfato simples, em toda área experimental. $\mathrm{O}$ início do período de diferimento ocorreu no dia $7 / 3 / 2006$, com a aplicação, em todas as parcelas, de $40 \mathrm{~kg} /$ ha de $\mathrm{N}$, em cobertura, no fim da tarde, e na forma de ureia. Em seguida, as parcelas foram irrigadas apenas para incorporação do adubo nitrogenado visando reduzir as possíveis perdas de nitrogênio por volatilização. Não se realizaram as avaliações no início do período de diferimento. Estas 
somente ocorreram no dia 1/7/2006, quando os perfilhos vegetativos e reprodutivos foram caracterizados nos pastos diferidos.

Determinou-se a densidade populacional de perfilhos por meio da colheita, com corte ao nível do solo de todos os perfilhos contidos no interior de um quadrado de 0,25 m de lado, em duas amostras por parcela. Esses perfilhos foram separados e quantificados em perfilhos vegetativos e reprodutivos. Classificaramse os perfilhos vivos que tinham a inflorescência visível como reprodutivos e os vivos que não tinham a inflorescência visível como vegetativos.

TABELA 1. Médias mensais da temperatura média diária, insolação, precipitação pluvial total e evaporação total durante o período de janeiro a julho de 2006

\begin{tabular}{|c|c|c|c|c|}
\hline Mês & $\begin{array}{l}\text { Temperatura média do ar } \\
\qquad\left({ }^{\circ} \mathrm{C}\right)\end{array}$ & Insolação (hora/dia) & Precipitação pluvial (mm) & Evaporação (mm) \\
\hline Janeiro & 23,1 & 7,2 & 180,0 & 108,6 \\
\hline Fevereiro & 23,6 & 9,4 & 84,8 & 82,4 \\
\hline Março & 22,6 & 5,6 & 186,5 & 62,5 \\
\hline Abril & 20,7 & 5,3 & 56,0 & 56,8 \\
\hline Maio & 17,2 & 5,3 & 6,4 & 59,4 \\
\hline Junho & 16,1 & 5,1 & 21,0 & 64,4 \\
\hline Julho & 15,7 & 6,5 & 6,3 & 79,0 \\
\hline
\end{tabular}

Fonte: Estação Meteorológica da Universidade Federal de Viçosa.

Posteriormente à amostragem para avaliação da densidade populacional de perfilhos, colheram-se, em cada parcela, amostras constituídas de 50 perfilhos de cada categoria. Essas amostras foram separadas em lâmina foliar viva, lâmina foliar morta e colmo vivo. Incorporou-se a região da lâmina foliar que não apresentava sinais de senescência (órgão de cor verde) à fração lâmina foliar verde. A região da lâmina foliar com amarelecimento e/ou necrosamento do órgão foi incorporada à fração lâmina foliar morta. Adicionou-se a bainha foliar viva, que se encontrava firmemente aderida ao colmo, na fração colmo vivo. As subamostras dos componentes morfológicos de cada categoria de perfilho foram acondicionadas em sacos de papel identificados. Estes foram levados à estufa de ventilação forçada, a $65^{\circ} \mathrm{C}$, por 72 horas e, em seguida, pesados. Com esses dados, calcularam-se o percentual dos componentes morfológicos e o peso unitário de cada categoria de perfilho.

As características estruturais foram avaliadas em dez perfilhos de cada categoria por parcela. Nestes perfilhos, quantificaram-se o comprimento do pseudocolmo e número de folhas vivas e mortas. O comprimento do pseudocolmo foi mensurado desde o nível do solo até a lígula da folha mais velha completamente expandida. As folhas vivas consistiram de folhas em expansão e expandidas. Classificaram-se apenas as folhas que possuíam mais de $50 \%$ da lâmina foliar senescente como mortas.

Em cada característica avaliada, procedeu-se à variância e, posteriormente, à análise de regressão para expressar os efeitos das parcelas (períodos de diferimento) dentro das subparcelas (categoria de perfilhos), cujo maior modelo de superfície de resposta em função das médias dos tratamentos foi o seguinte:

$$
\begin{aligned}
& \mathrm{Y}_{\mathrm{i}}=\beta_{0}+\beta_{1} \mathrm{X}_{\mathrm{i}}+\mathrm{e}_{\mathrm{i}}, \\
& \text { em que: } \\
& \mathrm{Y}_{\mathrm{i}}=\text { variável resposta; } \\
& \mathrm{X}_{\mathrm{i}}=\text { variável independente; } \\
& \beta_{0}, \beta_{1}=\text { parâmetros a serem estimados; } \\
& \mathrm{e}_{\mathrm{i}}=\text { erro experimental; }
\end{aligned}
$$

Os níveis dos fatores qualitativos (subparcelas) foram comparados dentro de cada nível dos fatores quantitativos (parcelas) pelo teste F. Realizaram-se todas as análises estatísticas por meio do Sistema para Análises Estatísticas (SAEG), versão 8.1 (UNIVERSIDADE FEDERAL DE VIÇOSA, 2003), adotando-se o nível de significância de até $10 \%$ de probabilidade. 


\section{RESULTADOS E DISCUSSÃO}

O período de diferimento não influenciou $(\mathrm{P}>0,10)$ o número de perfilhos vegetativos $(\mathrm{PV}) \mathrm{em}$ pastos diferidos de capim-braquiária (Tabela 2). Esse resultado não era esperado, uma vez que o maior sombreamento na base das plantas, comum em pastos sob maior período de diferimento, inibe o surgimento de novos perfilhos. Além disso, em condições de sombreamento, em geral, ocorre morte dos PV mais jovens e de menor tamanho, em virtude da competição com os PV de maior tamanho. De fato, em trabalho realizado por SANTOS et al. (2009a), o número de PV reduziu com o aumento da duração do período de diferimento da pastagem.

TABELA 2. Número e peso de perfilhos em pastos de capim-braquiária diferidos

\begin{tabular}{|c|c|c|c|c|c|}
\hline \multirow{2}{*}{ Perfilho } & \multicolumn{3}{|c|}{ Período de diferimento (dia) } & \multirow{2}{*}{ Equação de regreção } & \multirow{2}{*}{$\mathrm{R}^{2}$} \\
\hline & 73 & 95 & 116 & & \\
\hline & \multicolumn{5}{|c|}{ Perfilho/m² } \\
\hline Vegetativo & $1.291 \mathrm{a}$ & $1.605 \mathrm{a}$ & $1.024 \mathrm{a}$ & $\overline{\mathrm{Y}}=1.307$ & - \\
\hline \multirow[t]{2}{*}{ Reprodutivo } & $16 \mathrm{~b}$ & $21 \mathrm{~b}$ & $117 \mathrm{~b}$ & $\hat{Y}=-206,091+2,7686^{* * *} X$ & 0,79 \\
\hline & \multicolumn{5}{|c|}{ Peso $(g)$} \\
\hline Vegetativo & $0,31 \mathrm{~b}$ & $0,48 b$ & $0,95 \mathrm{a}$ & $\hat{\mathrm{Y}}=-0,9851+0,0166^{*} \mathrm{X}$ & 0,93 \\
\hline Reprodutivo & $0,76 \mathrm{a}$ & $0,81 \mathrm{a}$ & $1,05 \mathrm{a}$ & $\hat{\mathrm{Y}}=0,2382+0,0067 * * * \mathrm{X}$ & 0,88 \\
\hline
\end{tabular}

Médias seguidas pela mesma letra na coluna não diferem pelo teste de Tukey $(\mathrm{P}>0,05)$. ${ }^{*}$ Significativo pelo teste $\mathrm{t}(\mathrm{P}<0,01)$; *** Significativo pelo teste $\mathrm{t}(\mathrm{P}<0,10)$.

No entanto, houve $(\mathrm{P}<0,10)$ incremento linear no número de perfilhos reprodutivos (PR) com o período de diferimento (Tabela 2). Isso ocorreu porque pastos sob maior período de diferimento encontravam-se em estádio de maturidade mais avançado e, nessa situação, é natural o desenvolvimento dos PV em PR, conforme o ciclo fenológico normal da gramínea. TAMASSIA et al. (2001), avaliando o capim-de-rhodes em seis idades, também constataram incremento do número de inflorescência com o aumento na idade dos pastos. Adicionalmente, pastos de capim-braquiária submetidos aos menores períodos de diferimento foram diferidos mais tardiamente no ano, época em que já havia ocorrido o pico de florescimento mais intenso do capim-braquiária na região de Viçosa, MG, o que explica o menor número de PR nestes pastos. Esse pico de florescimento ocorreu nos pastos diferidos por maior período, justificando também seu maior número de PR.

O número médio de $\mathrm{PV}$ (1.307 perfilhos $/ \mathrm{m}^{2}$ ) foi maior $(\mathrm{P}<0,05)$ do que o de PR $\left(57\right.$ perfilhos $\left./ \mathrm{m}^{2}\right)$ em pastos de capim-braquiária diferidos por até 116 dias (Tabela 2). Esse mesmo padrão de resposta foi verificado por OLIVEIRA et al. (2007) em pastos diferidos de Brachiaria decumbens cv. Basilisk.

Em pastos diferidos por até 95 dias, perfilhos reprodutivos ( $\mathrm{PR}$ ) foram mais pesados $(\mathrm{P}<0,05)$ do que perfilhos vegetativos (PV), o que é explicado pelo inerente alongamento do colmo quando o PV desenvolve-se em PR. Contudo, nos pastos diferidos por 116 dias, não foi constatada diferença $(\mathrm{P}>0,10)$ entre os pesos de PV e PR (Tabela 2). Esse resultado se deve ao alongamento do colmo do PV em pastos sob maior período de diferimento, caracterizando uma resposta morfológica à condição de sombreamento, em que o índice de área foliar crítico já foi alcançado pelo dossel (KORTE et al., 1982). De fato, o alongamento do colmo ocorre já no estádio vegetativo em gramíneas do grupo fotossintético C4 (GOMIDE et al., 2003).

Os pesos dos perfilhos vegetativos $(\mathrm{P}<0,01)$ e reprodutivos $(\mathrm{P}<0,10)$ foram incrementados com o período de diferimento (Tabela 2). Isso ocorre, via de regra, em decorrência de perfilhos mais velhos possuírem maior massa em função do seu maior número de fitômeros.

A relação entre o número e o peso de perfilhos vegetativos $(\mathrm{PV})$ foi linear e negativa $(\mathrm{P}<0,10)$ (Figura 
1). Pastos diferidos por maior período apresentaram menor densidade populacional de PV maiores, ao contrário daqueles pastos diferidos por menor período, em que se observou maior número de $\mathrm{PV}$ pequenos. Essa resposta está de acordo com a lei de autocompensação entre tamanho e densidade de perfilhos (YODA et al., 1963).

De outra forma, a relação entre o número e o peso de perfilhos reprodutivos (PR) foi linear e positiva $(\mathrm{P}<0,10)$ (Figura 1). Em pastagens diferidas, o maior período de diferimento resulta em maior número de PR (Tabela 2), conforme já explicado. Nessa condição, também se verifica maior peso dos PR na medida em que estes foram originados de perfilhos vegetativos de maior tamanho.

As características estruturais dos perfilhos foram alteradas pelo período de diferimento da pastagem (Tabela 3). Nas duas categorias de perfilhos verificou-se incremento linear $(\mathrm{P}<0,01$ e $\mathrm{P}<0,10)$ do comprimento do pseudocolmo em função do período de diferimento da pastagem. Os pastos sob maior período de diferimento possuem perfilhos mais pesados (Tabela 2), que requerem colmo mais desenvolvido para sustentar seu peso. Aliás, a própria elevação no comprimento do pseudocolmo é um dos determinantes do maior peso dos perfilhos. SBRISSIA \& DA SILVA (2008) também verificaram que pastos de $B$. brizantha $\mathrm{cv}$. Marandu sob lotação contínua possuíram perfilhos maiores e com menor relação folha/colmo quando a altura média dos pastos incrementou de 10 para $40 \mathrm{~cm}$. Esse maior comprimento do pseudocolmo dos perfilhos ocorreu porque, provavelmente, houve competição por luz nos pastos diferidos por maior período. Nessa condição, ocorre um rearranjo na estrutura do pasto, cujas lâminas foliares se tornam mais espaçadas e eretas e, por conseguinte, melhoram o ambiente luminoso no interior do dossel (GOMIDE et al., 2003).
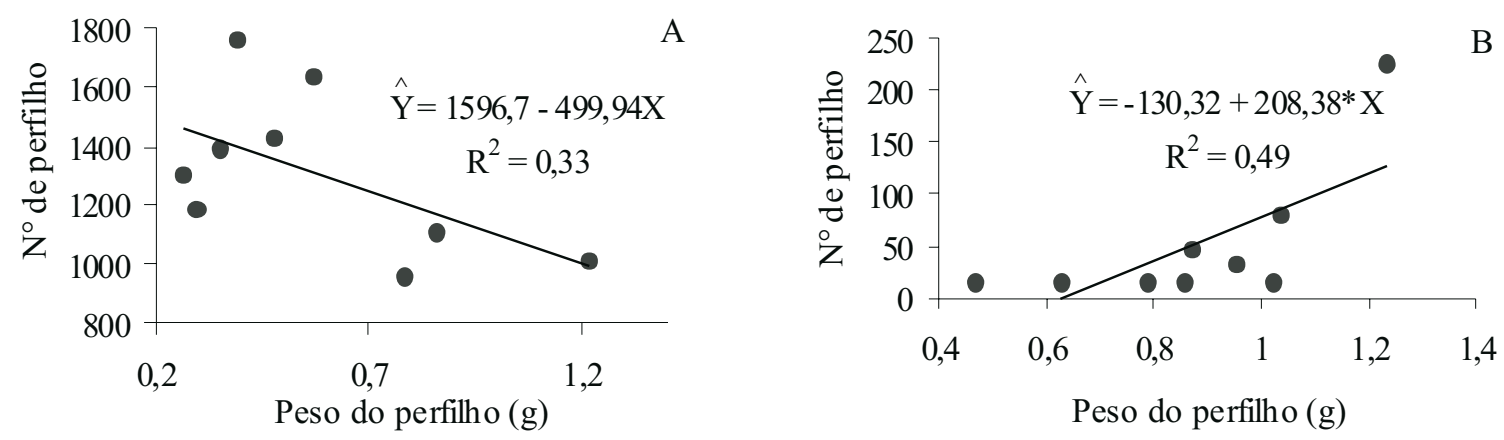

FIGURA 1. Relação entre número e peso de perfilhos vegetativos (A) e reprodutivos (B) em pastos diferidos de capim-braquiária * Significativo pelo teste $\mathrm{t}(\mathrm{P}<0,01)$.

TABELA 3. Características estruturais de perfilhos em pastos de capim-braquiária diferidos

\begin{tabular}{|c|c|c|c|c|c|}
\hline \multirow{2}{*}{ Perfilho } & \multicolumn{3}{|c|}{ Período de diferimento (dia) } & \multirow{2}{*}{ Equação de regreção } & \multirow{2}{*}{$\mathrm{R}^{2}$} \\
\hline & 73 & 95 & 116 & & \\
\hline & \multicolumn{5}{|c|}{ Comprimento do pseudocolmo $(\mathrm{cm})$} \\
\hline Vegetativo & $15,95 \mathrm{~b}$ & $25,30 \mathrm{~b}$ & $49,07 \mathrm{~b}$ & $\hat{\mathrm{Y}}=-42,549+0,7675^{*} \mathrm{X}$ & 0,94 \\
\hline \multirow[t]{2}{*}{ Reprodutivo } & $43,81 \mathrm{a}$ & 63,07 a & $78,00 \mathrm{a}$ & $\hat{\mathrm{Y}}=-13,705+0,7958 * * * \mathrm{X}$ & 0,99 \\
\hline & \multicolumn{5}{|c|}{ Número de folhas mortas } \\
\hline Vegetativo & $2,33 \mathrm{~b}$ & $2,80 \mathrm{~b}$ & $2,80 \mathrm{~b}$ & $\overline{\mathrm{Y}}=2,53$ & \\
\hline \multirow[t]{2}{*}{ Reprodutivo } & $4,33 \mathrm{a}$ & $4,67 \mathrm{a}$ & $4,73 \mathrm{a}$ & $\hat{\mathrm{Y}}=4,00461+0,00699 * \mathrm{X}$ & 0,97 \\
\hline & \multicolumn{5}{|c|}{ Número de folhas vivas } \\
\hline Vegetativo & $4,53 \mathrm{a}$ & $4,13 \mathrm{a}$ & $4,80 \mathrm{a}$ & $\overline{\mathrm{Y}}=4,57$ & - \\
\hline Reprodutivo & $2,53 \mathrm{~b}$ & $2,93 \mathrm{~b}$ & $2,73 \mathrm{~b}$ & $\bar{Y}=2,60$ & - \\
\hline
\end{tabular}

Médias seguidas pela mesma letra na coluna não diferem pelo teste de Tukey $(\mathrm{P}>0,05)$. ${ }^{*}$ Significativo pelo teste $\mathrm{t}(\mathrm{P}<0,01)$; *** Significativo pelo teste $\mathrm{t}(\mathrm{P}<0,10)$. 
O alongamento do pseudocolmo expõe as folhas mais novas na parte superior do dossel, porém resulta no sombreamento daquelas localizadas na parte inferior do pasto que, dessa maneira, senescem (LEMAIRE, 2001). Esse fato explica a elevação $(\mathrm{P}<0,01)$ do número de folhas mortas (NFM) nos perfilhos reprodutivos em pastos sob maior período de diferimento (Tabela 3). Nos perfilhos vegetativos, não foi observada $(\mathrm{P}>0,10)$ essa mesma resposta, o que foi inesperado. No entanto, o número de folhas vivas (NFV) por perfilho não foi incrementado $(\mathrm{P}>0,10)$ pelo período de diferimento (Tabela 3). Realmente, parece que o NFV por perfilho é uma característica genotípica bastante estável (FAGUNDES et al., 2006).

Pela comparação entre perfilhos vegetativos e reprodutivos, constatou-se que os perfilhos reprodutivos possuíram $(\mathrm{P}<0,05)$ maior comprimento do pseudocolmo, maior NFM e menor NFV (Tabela 3). Geralmente, perfilhos reprodutivos são de idade mais avançada e possuem maior número de fitômeros, cujas folhas encontram-se mortas em muitos deles. Ademais, o maior comprimento do pseudocolmo é característica inerente ao perfilho reprodutivo, conforme já explicitado.

As modificações nas características estruturais dos perfilhos vegetativos (PV) resultaram em mudanças na sua composição morfológica (Tabela 4). O período de diferimento incrementou o percentual de colmo $(\mathrm{P}<0,05)$ e diminuiu $(\mathrm{P}<0,10)$ o percentual de folha viva nos PV. Essas alterações são decorrentes do aumento no comprimento do pseudocolmo e manutenção do NFV nos perfilhos vegetativos (Tabela 3). Nestes, o percentual de folha morta não foi influenciado $(\mathrm{P}>0,10)$ pelo período de diferimento. Com relação aos perfilhos reprodutivos, não ocorreu resposta $(\mathrm{P}>0,10)$ com o período de diferimento na sua composição morfológica.

TABELA 4. Composição morfológica de perfilhos em pastos diferidos de capim-braquiária

\begin{tabular}{|c|c|c|c|c|c|}
\hline \multirow{2}{*}{ Perfilho } & \multicolumn{3}{|c|}{ Período de diferimento (dia) } & \multirow{2}{*}{ Equação de regreção } & \multirow{2}{*}{$\mathrm{R}^{2}$} \\
\hline & 73 & 95 & 116 & & \\
\hline & \multicolumn{5}{|c|}{ Colmo (\%) } \\
\hline Vegetativo & $36,74 \mathrm{~b}$ & $44,11 \mathrm{~b}$ & $55,11 \mathrm{~b}$ & $\hat{\mathrm{Y}}=1,39675+0,497 * * \mathrm{X}$ & 0,99 \\
\hline \multirow[t]{2}{*}{ Reprodutivo } & $60,35 \mathrm{a}$ & $60,88 \mathrm{a}$ & $61,86 \mathrm{a}$ & $\overline{\mathrm{Y}}=61,57$ & - \\
\hline & \multicolumn{5}{|c|}{ Folha viva $(\%)$} \\
\hline Vegetativo & $51,46 \mathrm{a}$ & $37,90 \mathrm{a}$ & 29,66 a & $\hat{\mathrm{Y}}=87,4745-0,510^{* * *} \mathrm{X}$ & 0,99 \\
\hline \multirow[t]{2}{*}{ Reprodutivo } & $14,43 \mathrm{~b}$ & $15,92 \mathrm{~b}$ & $8,04 \mathrm{~b}$ & $\bar{Y}=12,60$ & - \\
\hline & \multicolumn{5}{|c|}{ Folha morta $(\%)$} \\
\hline Vegetativo & $11,81 \mathrm{~b}$ & $17,99 \mathrm{~b}$ & $15,24 \mathrm{~b}$ & $\bar{Y}=15,16$ & - \\
\hline Reprodutivo & $25,22 \mathrm{a}$ & $23,21 \mathrm{a}$ & $30,10 \mathrm{a}$ & $\bar{Y}=25,83$ & - \\
\hline
\end{tabular}

Médias seguidas pela mesma letra na coluna não diferem pelo teste de Tukey $(\mathrm{P}>0,05)$.

** Significativo pelo teste $\mathrm{t}(\mathrm{P}<0,05)$;** Significativo pelo teste $\mathrm{t}(\mathrm{P}<0,10)$.

Na comparação entre o perfilho reprodutivo (PR) e o perfilho vegetativo (PV), a composição morfológica dos últimos foi mais influenciada pelo período de diferimento, demonstrando maior plasticidade fenotípica do $\mathrm{PV}$. Como era esperado, $\mathrm{PR}$ possuíram maiores $(\mathrm{P}<0,05)$ percentuais de colmo e folha morta, bem como menor percentual de folha viva $(\mathrm{P}<0,05)$ do que os $\mathrm{PV}$. Isso é devido ao maior comprimento do pseudocolmo, ao seu maior número de folha morta e ao menor número de folha verde observados no PR em relação ao PV (Tabela 3).
As modificações na composição morfológica de perfilhos individuais permitem explicar a estrutura menos favorável ao consumo do pasto diferido por maior período. Nesse contexto, como forma de melhorar a estrutura da forragem diferida, pode-se recomendar a redução no período de diferimento da pastagem, uma vez que essa ação de manejo melhora a composição morfológica dos perfilhos vegetativos, bem como reduz a densidade populacional de perfilhos reprodutivos. 


\section{CONCLUSÕES}

Em pastos de Brachiaria decumbens cv. Basilisk diferidos, perfilhos reprodutivos são mais pesados, possuem colmo mais comprido, maior número de folha morta, menor número de folha viva e pior composição morfológica que perfilhos vegetativos. A relação entre número e peso de perfilhos reprodutivos em pastos de Brachiaria decumbens cv. Basilisk diferidos por até 116 dias é positiva, e negativa para perfilhos vegetativos. O período de diferimento altera as características estruturais e piora a composição morfológica dos perfilhos em pastos diferidos de Brachiaria decumbens cv. Basilisk.

\section{REFERÊNCIAS}

EUCLIDES, V. P. B.; VALLE, C. B.; SILVA, J. M.; VIEIRA, A. Avaliação de forrageiras tropicais manejadas para produção de feno-em-pé. Pesquisa Agropecuária Brasileira, v. 25, n. 1, p. 63-68, 1990.

GOMIDE, J. A.; CÂNDIDO, M. J. D.; ALEXANDRINO, E. As interfaces solo, planta e animal da exploração da pastagem. In: FORRAGICULTURA E PASTAGENS: TEMAS EM EVIDÊNCIA - SUSTENTABILIDADE, 4., 2003, Lavras. Anais... Lavras: Editora da UFLA, 2003. p. 75-116.

HODGSON, J. Grazing management: science into practice. Essex: Longman Scientific \& Technical, 1990. 203 p.

KORTE, C. J.; WATKIN, B. R.; HARRIS, W. Use of residual leaf area index and light interception as criteria for spring-grazing management of ryegrass dominant pasture. New Zealand Journal of Agricultural Research, v. 25, p. 309-319, 1982.

LEMAIRE, G. Ecophisiology of grasslands: dynamic aspects of forage plant populations in grazed swards. In: GOMIDE, J. A.; MATTOS, W. R. S.; DA SILVA, S. C. (Eds.). INTERNATIONAL
GRASSLAND CONGRESS, 19., São Pedro, 2001. Proceedings... São Pedro. Disponível em: $<$ http://www.internationalgrasslands. org/publications > FEALQ, 2001. p. 29-37.

FAGUNDES, J. L.; FONSECA, D.M.; MISTURA, C. et al. Características morfogênicas e estruturais do capim-braquiária em pastagem adubada com nitrogênio avaliadas nas quatro estações do ano. Revista Brasileira de Zootecnia, v. 35, n. 1, p. 21-29, 2006.

SANTOS, M. E. R.; FONSECA, D. M.; EUCLIDES, V. P. B.; NASCIMENTO JR, D.; QUEIROZ, A. C.; RIBEIRO JR, J. I. Correlações, características estruturais e índice de tombamento de Brachiaria decumbens cv. Basilisk em pastagens diferidas. Revista Brasileira de Zootecnia, v. 38, n. 4, p. 626-634, 2009a.

SANTOS, M. E. R.; FONSECA, D. M.; BALBINO, E. M. Caracterização de perfilhos em pastos de capim-braquiária diferidos e adubados com nitrogênio. Revista Brasileira de Zootecnia, v. 38, n. 4, p. 643-649, 2009 b.

SANTOS, M. E. R.; FONSECA, D. M.; OLIVEIRA, I. M.; CASAGRANDE, D. R.; BALBINO, E. M.; FREITAS, F. P. Correlações entre número de perfilhos, índice de tombamento, massa dos componentes morfológicos e valor nutritivo da forragem em pastos diferidos de capim-braquiária. Revista Brasileira de Zootecnia, v. 39, n. 3, p. 487-483, 2010.

SBRISSIA, A. F.; DA SILVA, S. C. Compensação tamanho/densidade populacional de perfilhos em pastos de capim-marandu. Revista Brasileira de Zootecnia, v. 37, n. 1, p. 35-47, 2008.

UNIVERSIDADE FEDERAL DE VIÇOSA - UFV. SAEG sistema de análises estatísticas e genéticas. Versão 8.1. Viçosa, MG: UFV, 2003. (Apostila).

YODA, K.; KIRA, T.; OGAWA, R. HOZUMI, K. Intraspecific competition among higher plants. XI self-thinning in overcrowded pure stands under cultivated and natural conditions. Journal of Institute Polytechnics, Osaka City University, Series D, v. 14, p. 107-129, 1963.

Protocolado em: 21 out. 2008. Aceito em: 19 abr. 2010. 